\title{
A Correlative Cryo-Fluorescence and CryoSEM Approach for Visualizing Nanomaterials in Glioblastoma Tissue
}

\author{
$\underline{\text { Allessandra DiCorato }}^{1}$, Thomas O’Halloran $^{2}$, and Derk Joester ${ }^{1}$ \\ 1. Materials Science and Engineering, Northwestern University, Evanston, IL 60208, USA. \\ 2. Chemistry, Northwestern University, Evanston, IL 60208, USA.
}

A critical obstacle in the development of modern nanomaterials is the specific targeting of tumor cells and the penetration of nanoparticles into solid tumors. In order to expedite development, a thorough understanding of the barriers to delivery of nanomaterials is required. This demands visualizing the distribution of the nanomaterial throughout the tumor as well as the surrounding tissue. Furthermore, this visualization is necessary at multiple length scales, from the micrometer length scales of subcellular compartments and ultrastructural damage caused by the nanomaterials to the large scales of tumors themselves.

To address this need, we seek to establish a correlative cryo-imaging technique to spatially map the distribution of nanomaterials across cells and solid tumors. In particular, we seek to map the distribution of targeted liposomes ("nanobins") with stabilized $\mathrm{As}_{2} \mathrm{O}_{3}$ cores in glioblastoma tissue [1]. These nanomaterials have been visualized in both CryoTEM and CryoSEM (Fig. 1). Tumor cells are fluorescently labeled and are visualized along with unlabeled surrounding tissue. In order to meet the aforementioned length scale requirements, tumor tissues treated with targeted nanobins are examined at high resolution using CryoSEM and correlated with fluorescence microscopy to distinguish between tumor and healthy tissue.

To facilitate analysis in CryoSEM, we exploit CryoTIGM, or cryogenic triple ion gun milling, a new sample preparation strategy that employs triple argon beams to clear a flat plane biologically relevant in size [2]. This method offers superior surface flatness compared to conventional freeze fracture sample preparation for CryoSEM (Fig. 2). CryoTIGM also features higher throughput compared to similar techniques such as cryoFIB and can clear areas of $\sim 700,000 \mu \mathrm{m}^{2}$ in two hours [2]. Finally, CryoTIGM excels at revealing hard/soft interfaces. This technique is therefore ideal for visualizing nanomaterial cancer treatments in the context of cryo-preserved cells.

We have adapted our current ion milling setup to be compatible with a custom-built fluorescence microscope. This microscope offers superior resolution $(\sim 400 \mathrm{~nm})$ compared to a standard stereoscope and will allow us to greatly expedite analysis of fluorescently labeled tumor tissue within noncancerous tissue. Given the diffuse nature of glioblastoma tumors, noncancerous tissue is inevitably extracted alongside tumor tissue. Distinguishing between these tissues can be difficult without labeling. Fluorescent labeling offers perhaps the most efficient method to differentiate between samples containing tumor tissue and those that are wholly noncancerous. Furthermore, the ability to examine fluorescence in frozen specimens to correlate with CryoSEM would greatly aid an understanding of the nanomaterial distribution throughout cancerous and healthy tissue. In the most general sense, however, it allows one to correlate elemental information garnered from EDX and backscattered electron imaging in SEM with ultrastructural information accessible through fluorescence imaging [3].

References: 
[1] TV O'Halloran et al, Theranostics 3 (2013), p. 496.

[2] IYT Chang and D Joester, Microscopy and Microanalysis 21 (2015), p. 1616.

[3] The authors acknowledge funding from the NSF Major Research Instrumentation program (NSF MRI1229693) and the Northwestern University Materials Research Center (DMR-1121262). Research reported in this publication was supported by the National Institute of General Medical Sciences of the National Institutes of Health under Award Number T32GM105538. The content is solely the responsibility of the authors and does not necessarily represent the official views of the National Institutes of Health.

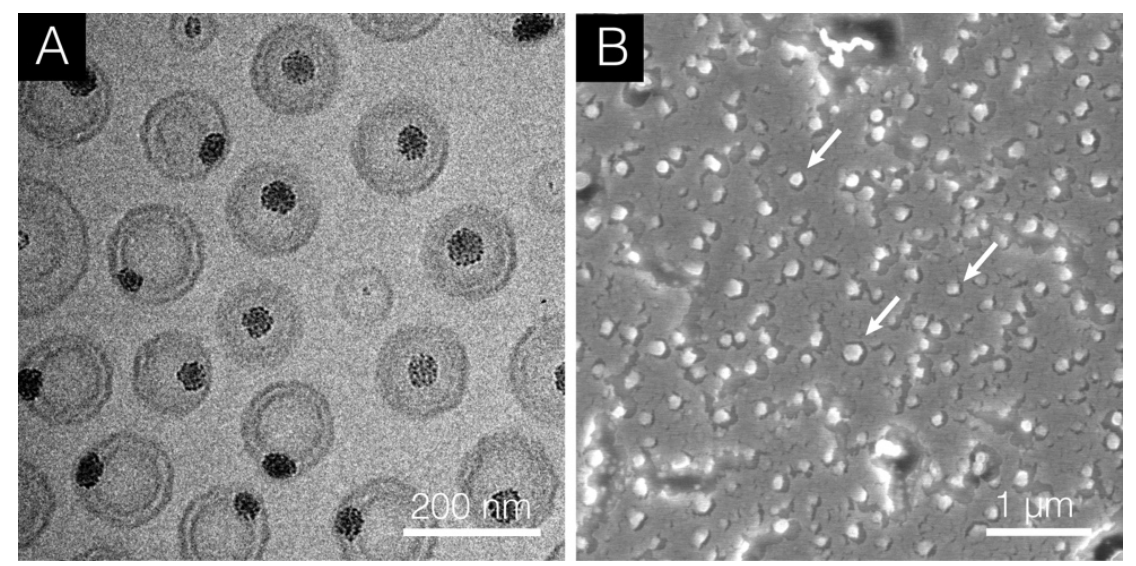

Figure 1. a) CryoTEM image of a suspension of liposomes with Pt- stabilized $\mathrm{As}_{2} \mathrm{O}_{3}$ cores. b) CryoSEM image of suspension of liposomes (white arrows). Image in a) courtesy of Eric Roth.
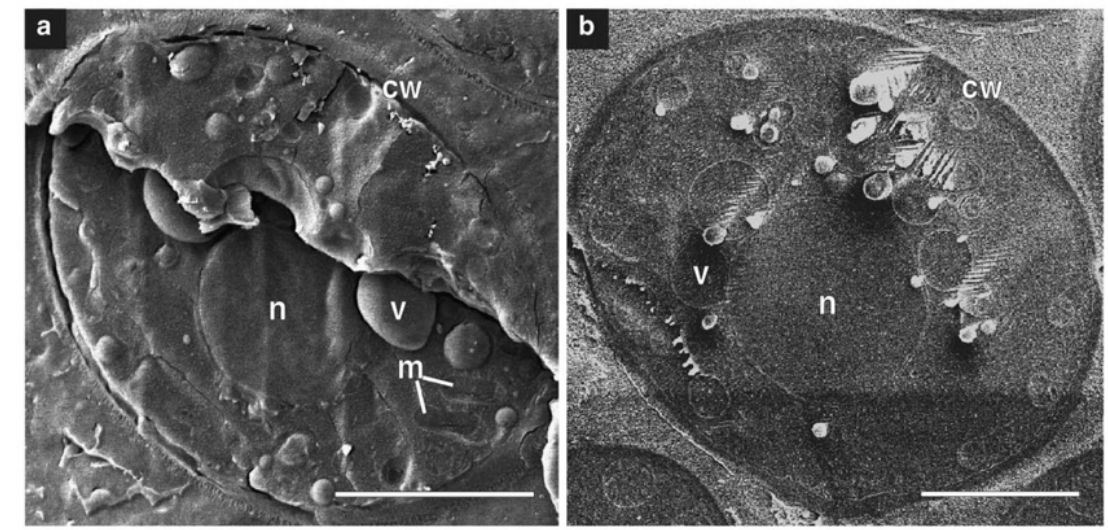

Figure 2. S. cerevisiae cells prepared for CryoSEM using a) the conventional freeze-fracture method, and b) CryoTIGM. Superior flatness and resolution of cellular ultrastructure is observed in $\mathrm{b}$. The cell wall $(\mathrm{cw})$, nucleus (n), mitochondria (m), and vesicles (v) are indicated. Scale bar is $2 \mu \mathrm{m}$. Figure adapted from [2]. 